DOI: $10.1515 /$ awutp-2015-0202

\title{
APPROXIMATE SOLUTION OF SCHRÖDINGER EQUATION WITH PSEUDO-GAUSSIAN POTENTIAL VIEWED AS A PERTURBATION
}

\author{
Theodor-Felix Iacob ${ }^{\mathrm{a}, *}$, Marina Lute ${ }^{\mathrm{b}, \dagger}$, Felix Iacob ${ }^{\mathrm{a}, \ddagger}$ \\ a West University of Timișoara \\ 300223 V. Pârvan Ave 4, Timisoara, Romania \\ b Politehnica University of Timisoara \\ 300223 Traian Lalescu 2, Timișoara Romania \\ * theodor.iacob95@e-uvt.ro, ${ }^{\dagger}$ marina.lute@ct.upt.ro, ${ }^{\ddagger}$ felix.iacob@e-uvt.ro
}

\begin{abstract}
Article info
Received: 15.10 .2015

Accepted: 16.11 .2015
\end{abstract}

Keywords: Quantum mechanics, Gaussian potential, perturbation theory, Energy levels.

PACS: 03.65.Ta

\begin{abstract}
We consider the Schrödinger equation with pseudo-Gaussian potential and point out that it is basically made up by a term representing the harmonic oscillator potential and an additional term, which is actually a power series that converges rapidly. Based on this observation the system can be considered as a perturbation of harmonic oscillator. The perturbation method is used to approximate the energy levels of pseudoGaussian oscillator. The results are compared with those obtained in the analytic and numeric case.
\end{abstract}

\section{Introduction}

The solution of Schrödinger equation (SE) continues to attract interest of physicists and mathematicians. Solving this equation is not always a simply matter, thus different methods have been developed to solve this equation. SE can be solved exactly for a small number of potentials, which are nevertheless of extreme theoretical importance to develop theoretical models. Applied physics faces the reality of complicated potentials, which cannot be solved exactly, for these SE have to be solved in suitable ways more or less exactly. In addition to such models there is a wide class of partially solvable models, which admit exact solutions only for some states or particular values of the parameters in the Hamiltonian. In the last years there has been a vast literature about such problems. Among these we mention anharmonic oscillators with either polynomial [1, 2] or rational [3] potentials, and perturbed Coulomb [4] and Laurent-like [5] potentials. Although in such cases one obtains some eigenvalues and eigenfunctions exactly, it is not always a 
simple matter to obtain normalization factors, and thereby expectation values, in closed form [6]. Partially solvable models are also a useful benchmark to test the accuracy of approximate methods.

The exact solution of Schrödinger equation for the pseudo-Gaussian potential was presented in [7]. It was found that this potential belongs to the class of partially solvable models. Furthermore it was shown that the pseudo-Gaussian potential expands into a polynomial, systems known in literature as quasi-exact systems.

In this paper, based on an observation upon the pseudo-Gaussian Hamiltonian, we apply Rayleigh-Schrödinger perturbation theory to calculate the eigenvalues and eigenfunctions. The model with this potential was named pseudo-Gaussian oscillator (PGO) $[8,9]$. Using perturbation theory, one can use the known solutions of simple Hamiltonians to generate solutions for a range of more complicated systems. In our case the PGO potential can be written as a sum between harmonic oscillator (HO) potential and a power series, considered as a perturbaton.

\section{The physical model}

We will present the physical model as it was introduced in $[9,10]$ with the coefficients $C_{k}$. This model have been used to find energy levels for a system described by KleinGordon equation. Let us consider the radial part of the three-dimensional Schrödinger's time-independent equation, $\mathbf{H} \psi=E \psi$, the square-integrable complex functions $\psi$ of real variable are called eigenfunctions and the numbers $E$ are called eigenvalues of the energy. The Hamiltonian operator $\mathbf{H}$, acting on the space of eigenfunctions, given in atomic units, $\mathbf{H}=-\frac{1}{2} \Delta+V(r)$, introduces the central real valued potential $V(r)$ on Euclidean real space with spherical coordinates as:

$$
V_{\lambda, \mu}^{s}(r)=\left(\lambda+\sum_{k=1}^{s} C_{k} r^{2 k}\right) \exp \left(-\mu r^{2}\right)
$$

having the coefficients $C_{k}$,

$$
C_{k}=\frac{(\lambda+k) \mu^{k}}{k !}
$$

The properties of this model are completely determined by the dimensionless parameters $\lambda \in \mathbb{R}, \mu \in \mathbb{R}_{+}$and the positive integer $s=1,2, \ldots$, named the order of PGO. We note that the genuine Gaussian potential corresponding to the order $s=0$ is not included in this family. The potentials defined by the eqs. (1) and (2) have the remarkable property to approach to the HO potential when $r \rightarrow 0$ together with Gaussian asymptotic behavior, i.e. $\lim _{r \rightarrow \infty} V_{\lambda, \mu}^{s}(r)=0$. We also have to notice that, for each order, $s$, the Taylor expansion of these potentials does not have terms proportional with $r^{4}, r^{6}, \ldots, r^{2 s}$.

$$
V_{\lambda, \mu}^{s}(r)=\lambda+\mu r^{2}+O\left(r^{2 s+2}\right)
$$


In figure (1) it is shown the graph of both PGO and $\mathrm{HO}$, one can see their similar shape in a vicinity of origin and the Gaussian asymptotic behavior of PGO beside HO, which goes to infinity.

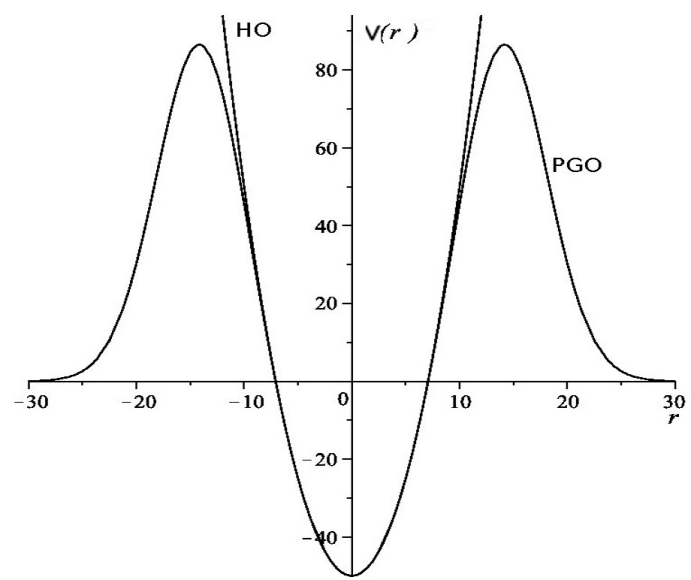

Figure 1: The pseudo-Gaussian oscillator potential graph $(s=3)$ compared with harmonic oscillator potential one.

Taking into consideration that $l(l+1)$ represents the eigenvalue of the square of the angular-momentum operator $\mathbf{L}^{2}$, the radial part of the Schrödinger equation for the stationary states can be written as:

$$
\left[\frac{d^{2}}{d r^{2}}+\frac{(2)}{r} \frac{d}{d r}+(E-V(r))-\frac{l(l+1)}{r^{2}}\right] \psi(r)=0
$$

with $\psi(r)$ the radial wave-function. Considering the potential (1) we can study the radial three dimensional Schrödinger eigenvalue problem. We can eliminate the first derivative by setting

$$
\psi(r) \equiv r^{-1} R(r)
$$

and (4) becomes:

$$
\left[\frac{d^{2}}{d r^{2}}+(E-V(r))-\frac{l(l+1)}{r^{2}}\right] R(r)=0 .
$$

We can calculate the series (3) of potential (1) with coefficients (2), and after some algebra we get the form:

$$
V_{\lambda, \mu}^{s}(r)=\lambda+\mu r^{2}+\sum_{k=s+1} \hat{C}_{k} r^{2 k}
$$

with coefficients $\hat{C}_{k}$ coming from the Taylor expansion and having the following form [7]:

$$
\hat{C}_{k} \propto(-1)^{s+k}\left(\frac{1}{(k-1) !}+\frac{\lambda}{k !}\right)(k-s)^{\frac{k}{2}}
$$

The potential (1) with terms grouped as in (7), allows us to recognize that it is basically made up by a term representing the HO potential $V_{H O}=\lambda+r^{2}$, with $\lambda$ an arbitrary energy level and an additional term, which is actually a power series, $V_{i n t}=\sum_{k=s+1} \hat{C}_{n} r^{2 k}$. 
The analytical solutions of PGO system, where introduced in [7] and the values of energy levels were calculated. It was also shown that the PGO potentials belong to the class of partially solvable models, so it is a useful benchmark to test the accuracy of the quasi-exact approach with an approximate methods. In this way we propose the Raleigh-Schrödinger perturbation theory to approximate the eigenvalues. We want to see how different particular restriction on the quasi-exact approach affects on the eigenvalues by comparing them with the results obtained both by perturbative and numeric method. This approach is not singular, for example in the paper [11], the solutions of the Schrödinger equation for harmonic oscillator with a singular perturbation are presented.

\section{Perturbed PGO system.}

In our case the perturbation theory deals with the eigenvalue problem of the Schrödinger equation $\mathbf{H} \psi=E \psi$, where $\mathbf{H}=\mathbf{H}_{0}+\mathbf{H}^{\prime}$ contains a zeroth order part $\mathbf{H}_{0}$ and the perturbation $\mathbf{H}^{\prime}$. The standard perturbation procedure gives that expanding strictly in powers of the smallness of $\mathbf{H}^{\prime}$, we have the matrix solution:

$$
\begin{aligned}
E & =E^{(O)}+E^{(1)}+E^{(2)}+\ldots \\
\psi & =\psi^{(O)}+\psi^{(1)}+\ldots \\
E_{n}^{(O)} \mid n> & =\mathbf{H}_{0} \mid n> \\
E_{n}^{(1)} & =<n\left|\mathbf{H}^{\prime}\right| n> \\
\psi_{n}^{(1)} & =\sum_{m} \mid m>\frac{<m\left|\mathbf{H}^{\prime}\right| n>}{E_{n}^{(O)}-E_{m}^{(O)}}
\end{aligned}
$$

where $\mid n>$ are the complete set of eigenstates $\psi_{n}^{(0)}$ of $\mathbf{H}_{0}$. The expression for $E_{n}^{(1)}$ is simple enough, just a single integral needs to be done in order to find the first order perturbation energy. Unfortunately the expression for $E_{n}^{(2)}$ is complicated, in order to find the second order perturbation energy we have to solve an infinite sum over integrals

$$
E_{n}^{(2)} \mid n>=\sum_{m} \frac{<n\left|\mathbf{H}^{\prime}\right| m><m\left|\mathbf{H}^{\prime}\right| n>}{E_{n}^{(O)}-E_{m}^{(O)}}
$$

that one seldom can evaluate beyond first-order energy shift without making some gross approximations. This made us to stop to the first order perturbations, but fortunately we can add a correction to the second order one as is stated in [12]:

$$
E_{n}^{(2)}=\int \psi_{n}^{(0) *}\left(\mathbf{H}^{\prime}-E_{n}^{(1)}\right) \psi_{n}^{(1)} d r
$$

With this correction we the applied perturbation theory to PGO system, introduced in previous section. Let $\mathcal{D}$ denote the differential operator on the Hilbert space, the unperturbed Hamiltonian writes: $\mathbf{H}_{0}=\mathcal{D}^{2}+\left(\lambda+\mu r^{2}\right) \times \mathbf{I}$ and for perturbation we have 
the Hamiltonian :

$$
\mathbf{H}^{\prime}=\sum_{k=s+1} \hat{C}_{n} r^{2 k} \times \mathbf{I}
$$

where coefficients $\hat{C}_{n}$ were introduced by rel. (8).

Precautions have to be taken because it is a degenerate system. In this case it is possible, that the denominator $E_{n}^{(O)}-E_{m}^{(O)}$ of relations (10), (11) and so on, to become zero. In this sense we consider variation of principal quantum number $n$ keeping $l=0$. According to the aufbau principle corresponding to free atom there are no crossing energy states, thus a state with angular momentum $(l+1)$ is not more strongly bound than the one with $l$. The accidental degeneracy lifting along with the crossing energy states was studied by one of the authors in the case of confinement [10]. Another fact is that small perturbation can determine major changes in the form of the wave function. This can be somehow avoided by taking combinations that most closely resemble the final form of the wave functions. This is possible due to the fact that any linear combination of degenerate eigenfunctions is also an eigenfunction of the Hamiltonians. Continuing in the previous manner, keeping $l=0$, we can apply the theory for the non-degenerate system. Otherwise we have to consider all degenerate eigenstates of Hamiltonian and extract the energy values by solving the secular determinant.

Finally we suppose that by perturbation there are no transitions to continuum states. With this observations and restriction we proceed to calculate the energy levels.

To calculate the first order correction to the energy, we need to evaluate eq. (9) this is the integral:

$$
E_{n}^{(1)}=\int R_{n}(r)\left[\mathbf{H}^{\prime} R_{n}(r)\right] d r
$$

the general solution of radial $\mathrm{HO}$ is

$$
\psi_{n l m}(r, \theta, \phi)=N_{n l} r^{l} e^{-\nu r^{2}} L_{n}^{\left(l+\frac{1}{2}\right)}\left(2 \nu r^{2}\right) Y_{l m}(\theta, \phi)
$$

so the radial part reads:

$$
R_{n}(r)=N_{n l} r^{l} e^{-\nu r^{2}} L_{n}^{\left(l+\frac{1}{2}\right)}\left(2 \nu r^{2}\right)
$$

where $\nu \equiv \frac{\mu}{2 \hbar}$;

$$
N_{n l}=\sqrt{\sqrt{\frac{2 \nu^{3}}{\pi}} \frac{2^{n+2 l+3} n ! \nu^{l}}{(2 n+2 l+1) ! !}}
$$

is a normalization constant; and $L_{n}{ }^{\left(l+\frac{1}{2}\right)}\left(2 \nu r^{2}\right)$ are generalized Laguerre polynomials. The integral (14) can be written now as:

$$
E_{n}^{(1)}=N_{n l}^{2} \int_{\mathcal{D}} e^{-2 \nu r^{2}} L_{n}{ }^{\left(l+\frac{1}{2}\right)}\left[\mathbf{H}^{\prime} L_{n}{ }^{\left(l+\frac{1}{2}\right)}\right] d r
$$

We have evaluated this integral numerically,in the case $N=7, \lambda=-5.6, \mu=0.2$ and $l=0$. The obtained values are written in the table (1): 
Table 1: Calculated perturbative values.

\begin{tabular}{|c|c|c|c|c|c|c|c|c|c|}
\hline $\mathrm{n}$ & 0 & 1 & 2 & 3 & 4 & 5 & 6 & 7 & 8 \\
$\left\langle n\left|\mathbf{H}^{\prime}\right| n>\right.$ & 0.481 & 0.365 & 0.027 & 0.345 & 1.022 & 1.585 & 1.709 & 1.844 & -2.816 \\
\hline
\end{tabular}

Here we are at the point to remind that, according with the definition of PGO potential, the terms proportional with $r^{4}, r^{6}, \ldots, r^{2 s}$ vanish from the power series. In this way the first coefficient $\hat{C}_{k} \quad k=s+1$ of the power series has always a negative value as rel. (8) reveals. The calculated results are presented in figure (2) along with the data obtained in the exact case [7] and data obtained in the generating functional approach [8] but recalculated with the coefficients $C_{k}(2)$, introduced in [9], which makes the PGO potential shallowest, so in consequence the energy levels will be lifted from the case. presented in [8]

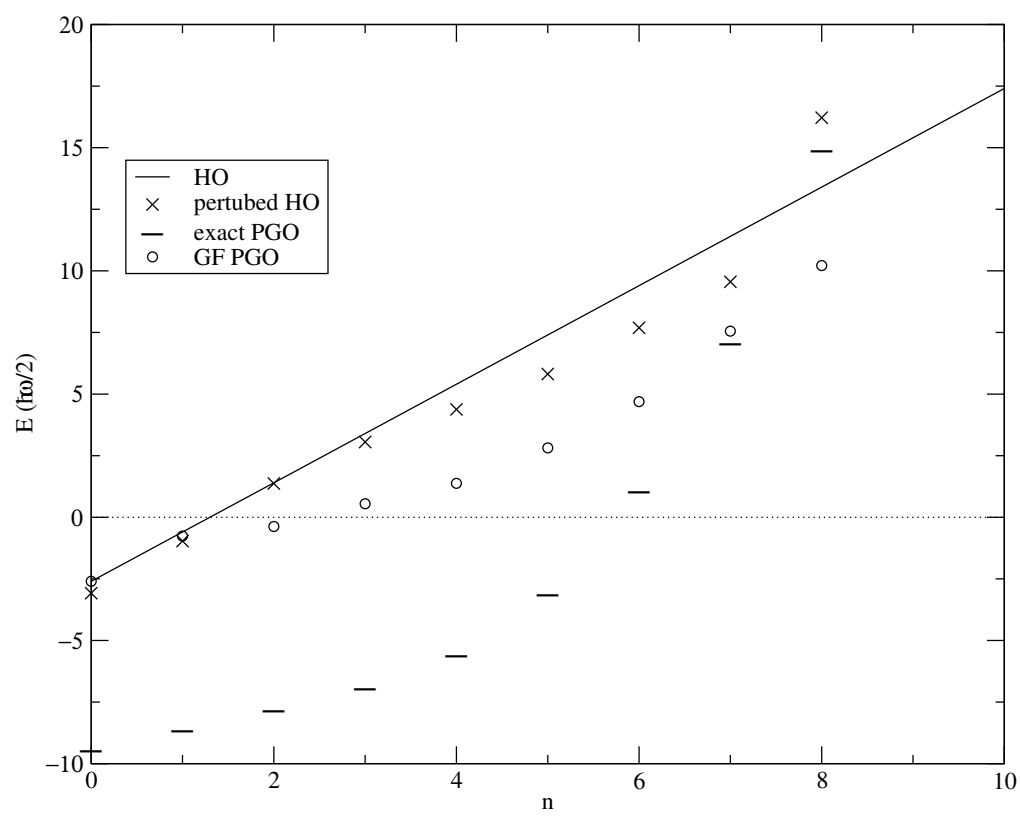

Figure 2: The eigenvalues given by Rayleigh-Schrödinger perturbation theory.

In conclusion we have explored the technique of evaluating energy levels of PGO by perturbation theory, from the point of view of Schrödinger's differential equation. The method seems adaptable to the problem, just only in some cases. In our approach we are based on the comparison with other methods and the shape of polynomial interpolating the energy poles is somehow preserved for all methods. We can see that even the generating functional method [8] is more accurate than the perturbative one. The values obtained by perturbation method are the worst approximation by any other method, however the arrangement of energy levels is preserved. This means the tendency of energy levels to be more distant to each other and under HO levels as approaching to zero level is maintained.

\section{References}

[1] R. S. Kaushal, Phys. Lett. A 142 (1989) 57.

[2] H. H. Aly and A. O. Barut, Phys. Lett. A 145 (1990) 299. 
[3] P. Roy and R. Roychoudhury, J. Phys. A, 23 (1990) 1657.

[4] R. Adhikari. R. Dutt and Y.P. Varshni, Phys. Lett. A 141,(1989) 1.

[5] L. D. Salem, R. Montemayor, Phys. Rev. A 43 (1991) 1169.

[6] M. Puta, F. Iacob, Ann. Univ. Timisoara XXXIX (2001) 385.

[7] F. Iacob, M. Lute, J. Math. Phys., 56, 12, (2015)

[8] I. I. Cotaescu, P. Gravila, M. Paulescu, Int. J. Mod. Phys. C 19 (2008) 1607.

[9] F. Iacob, Phys. Lett. A 374, 11-12, 1332-1335 (2010).

[10] F. Iacob, Centr. Eur. J. Phys. 12 (2014) 628.

[11] F. M. Fernandez, Phy. Lett. A 160 (1991) 511.

[12] C. Schwartz, Ann. Phys. 2 (1959) 156.

[13] L. E. Ballentine, Quantum Mechanics: A Modern Development (WS Publ. London, 2000). 